\title{
Discussion
}

L. Gouguenheim: I wish also to comment that a similar lab was successfully given by L. Celnikier during a summer school for teachers. The report was published in the proceedings of the TARBES Summer School (1978, L. Celnikier, Editor, Publication de l'Observatoire de Paris).

J. Fierro: Covering the sandbox with colored powder or cornstarch will show ejecta and rays for the crater experiment you described.

J.V. Feitzinger: In the book Astronomie Experimentelle (Experimental Astronomy) by J. Meurers, about 1955, you will find detailed descriptions of experiments on cratering. The production of central bulges is described.

W. Bisard: Yes, thank you.

J.-C. Pecker: All these types of experiments are good inasmuch as they help students to make actual measurements, build diagrams, and understand the physical processes that may happen in the universe. But these is a danger of misconceptions: for example, some young people might think that large craters on the moon are created by objects that are coming from far away, small ones by objects that are coming from the vicinity.... So teachers should heavily comment about the difficulty of generalization, and of extrapolation of classroom experiments to the real astronomical world.

O. Gingerich: Can you get elliptical craters? How do you measure the sideways component?

W. Bisard: Yes, especially with very oblique impacts. The astronomical craters are circular and are caused by the explosion of superheated gases at the impact site. No evidence can be found of initial sideways velocities of astronomical impacts.

\section{LABORATORY ACTIVITIES IN THE TEACHING OF ASTRONOMY}

\author{
Wayne Osborn \\ Central Michigan University, Mt. Pleasant, Michigan 48859, U.S.A
}

Astronomy is one of the most popular of the sciences. That this is so can be seen in the frequent news articles about astronomical discoveries and happenings, in the number of questions about the sky fielded by planetarium and observatory staff, and in the large turnouts for public observing sessions at observatories or astronomy 
club star parties.

Another reflection of astronomy's popularity is the fact that thousands of university students study the subject each year. Only a few of these are training to become professional astronomers. The vast majority of these students seek mainly to obtain a general background. An important subset of the latter group are those students preparing to teach in the schools and who have the need to understand, and be able to explain, common astronomical phenomena clearly. Those of us who teach introductory astronomy courses have an obligation to ensure that these prospective teachers are well trained.

Traditionally, astronomy courses consist of lectures and associated laboratory activities. The purpose of the lectures is to present and explain astronomical concepts and describe the various astronomical objects. Depending on the goals and backgrounds of the students, the level of lecture presentation ranges from purely descriptive to highly technical and quantitative.

It should go without saying that the laboratory exercises associated with an astronomy course also should depend on the type of students being served. Those intending to become professional astronomers must learn the techniques of observing and the methods of analysis of astrophysical data. Most astronomy educators would agree that in this case the activities should resemble true astronomical practice as closely as time, the available equipment, and the sophistication of the students permit. The chief difficulty encountered is having access to modern instrumentation, especially at less-developed institutions. The new "traveling telescope" project of IAU Commission 46 is a step toward solving this problem.

The laboratory exercises designed for professional training are, however, of little importance to the student with only a general interest in astronomy. For these students simple equipment is entirely suitable for the observing and measurement involved in laboratory work. The major concern is to select the most appropriate activities. I would argue that often little thought is given to this matter.

Traditionally the laboratory components of general astronomy courses stress activities that illustrate technical concepts. Examples are exercises on spectral classification, the H-R diagram, and the redshift-distance relation for galaxies (Hubble's Law). These activities reinforce material in the lecture course and provide the student with a feeling for how science is done, but they have little everyday practicality. Demonstrating the process of science is important, but the topics through which this is done need not be those of most interest to professional astronomers. Of more immediate and practical use to general interest students are exercises on common astronomical effects and on simple observing. Examples include activities that demonstrate the causes of day and night, the seasons, and lunar phases, and ones that involve constellation and planet identification and the use of a small telescope. Simple observing projects can be used as the basis for showing scientific methods.

Clear knowledge of the everyday astronomical phenomena is particularly important for school teachers because this is the astronomy that is required to be taught in schools. As an example, my home state of Michigan specifies the following seven topics as the astronomy that must be taught (grade level is in parentheses): 
the cause of day and night (4-6), the relationship between the earth and the moon (4-6), the motion of the earth and planets (4-6), major structures in the universe including the solar system (4-6), the phases of the moon (7-9), theoretical origins of the solar system and the universe (7-9), and astronomical equipment including telescopes (7-9).

At Central Michigan University we have developed a laboratory course for general astronomy students that is based almost entirely on practical and elementary concepts. Activities include learning to identify the prominent constellations, bright stars, and naked-eye planets; charting the motions of planets and seeing how this affects their visibility; observing and understanding diurnal and seasonal changes in the sky; knowing the phases of the moon, their times of visibility, and the cause; understanding the different types of eclipses; learning the components and the use of a small astronomical telescope; learning the use of star maps and the celestial globes; and viewing the sun, moon and planets. A copy of the laboratory manual may be obtained by contacting the author.

\title{
TEACHING OBSERVATIONAL ASTRONOMY AS A LABORATORY COURSE FOR NON-MAJORS
}

\author{
Yong H. Kim \\ Department of Astronomy, Geosciences \& Physics, Saddleback College, \\ Mission Viejo, California 92692, U.S.A.
}

\section{Introduction}

Since antiquity, doing astronomy means basically stepping outside, looking upward, and considering the widest environment. Thus any undergraduate astronomy program, no matter how diverse its course offering, is incomplete without observational astronomy. For example, some California community colleges offer several courses including such titles as "Man and the Cosmos," "Final Stellar States," "Astronomy Enrichment," and "Astronomical Myths, Mysteries \& Fallacies," but do not offer "Observational Astronomy." As a teaching astronomer, I question the wisdom and honesty of such practice of proliferation solely based on sensationalism. An introductory lecture course and an observational lab course must be the core of lower-division undergraduate astronomy education. Anything else, in my opinion, is peripheral.

This paper intends to address the importance of the teaching of observational astronomy as a liberal studies lab course. Instead of delineating specific course materials or instructional methods, I aim to discuss epistemological goals which, I 